\title{
Statistical properties of fracture in a random spring model
}

\author{
Phani Kumar V. V. Nukala \\ Computer Science and Mathematics Division, Oak Ridge National Laboratory, Oak Ridge, Tennessee 37831-6359, USA \\ Stefano Zapperi \\ INFM UdR Roma 1 and SMC, Dipartimento di Fisica, Università ”La Sapienza," P. le A. Moro 2, 00185 Roma, Italy \\ Srđan Šimunović \\ Computer Science and Mathematics Division, Oak Ridge National Laboratory, Oak Ridge, Tennessee 37831-6359, USA
}

(Received 3 February 2005; published 9 June 2005)

\begin{abstract}
Using large-scale numerical simulations, we analyze the statistical properties of fracture in the twodimensional random spring model and compare it with its scalar counterpart: the random fuse model. We first consider the process of crack localization measuring the evolution of damage as the external load is raised. We find that, as in the fuse model, damage is initially uniform and localizes at peak load. Scaling laws for the damage density, fracture strength, and avalanche distributions follow with slight variations the behavior observed in the random fuse model. We thus conclude that scalar models provide a faithful representation of the fracture properties of disordered systems.
\end{abstract}

DOI: 10.1103/PhysRevE.71.066106

PACS number(s): 46.50.+a, 64.60.Ak

\section{INTRODUCTION}

The statistical properties of fracture in disordered media represent an intriguing theoretical problem with important practical applications [1]. The presence of disorder naturally leads to statistical distributions of failure stresses, accumulated damage, acoustic activity, crack shapes, and so on. The application of a standard continuum description based on an elastic equation cannot capture the effect of fluctuations, and hence the effect of disorder has to be considered explicitly. A well established way to deal with this problem relies on lattice models, in which the medium is described by a discrete set of elastic bonds with randomly distributed failure thresholds [1]. In the simplest approximation of a scalar displacement, one recovers the random fuse model (RFM) where a lattice of fuses with random thresholds is subject to an increasing external current [2].

The RFM has been extensively investigated in the past 20 years, mainly using numerical simulations [1-8]. The type of behavior at macroscopic fracture is significantly influenced by the amount of disorder [3]. When the disorder is narrowly distributed, materials break down without significant precursors. In particular, if the threshold distribution does not extend to zero, the lattice breaks suddenly [3]. As the disorder increases, substantial damage is accumulated prior to failure and the dynamics resembles percolation. Indeed, in the limit of infinite disorder, the damage accumulation process can exactly be mapped onto a percolation problem [9]. It has been suggested that for strong, but finite, disorder, fracture should be interpreted as a first-order transition near a spinodal point [6]. In addition, the fracture of the RFM is preceded by avalanches of failure events $[6,10-12]$. These are reminiscent of the acoustic emission activity observed in experiments and their distribution follows a power law. Finally, the RFM has also been used to compute the fracture strength distribution and the related size effects [13-17].

Modeling the elastic medium using the RFM introduces drastic approximations in terms of the discretization process, quasistatic dynamics, and the scalar nature of the interactions. It is thus important to clarify if the observations made in the RFM carry over to more complex and realistic situations. In this paper, we address the problem of the scalar (electric) interactions of the RFM, by comparing it with a tensorial central force model, the random spring model (RSM) $[18,19]$. The model is a tensorial counterpart of the RFM: it has quasistatic dynamics, random thresholds, but fuses and currents are replaced by elastic springs and forces. Dynamic effects have been considered instead in Refs. $[20,21]$.

After discussing the model in Sec. II, we consider the typical statistical measures performed using the RSM: damage localization and average damage profiles are reported in Sec. III, while mean damage scaling and damage distributions are discussed in Secs. IV and V, respectively. In Secs. VI and VII, we discuss the fracture strength distribution and the size effect on the mean strength. The avalanche behavior is analyzed in Sec. VII and a summary is reported in Sec VIII. We have not analyzed the roughness of the final crack since in several instances the spring networks fail because of loss of rigidity.

\section{THE RANDOM SPRING MODEL}

In the RSM, the lattice is initially fully intact with bonds having the same stiffness, but the bond-breaking thresholds, $t$, are randomly distributed based on a threshold probability distribution, $p(t)$. The bond breaks irreversibly, whenever the force in the spring exceeds the breaking threshold force value, $t$, of the spring. Periodic boundary conditions are imposed in the horizontal direction and a constant unit displacement difference is applied between the top and the bottom of lattice system.

Numerically, a unit displacement, $\Delta=1$, is applied at the top of the lattice system and the equilibrium equations are 


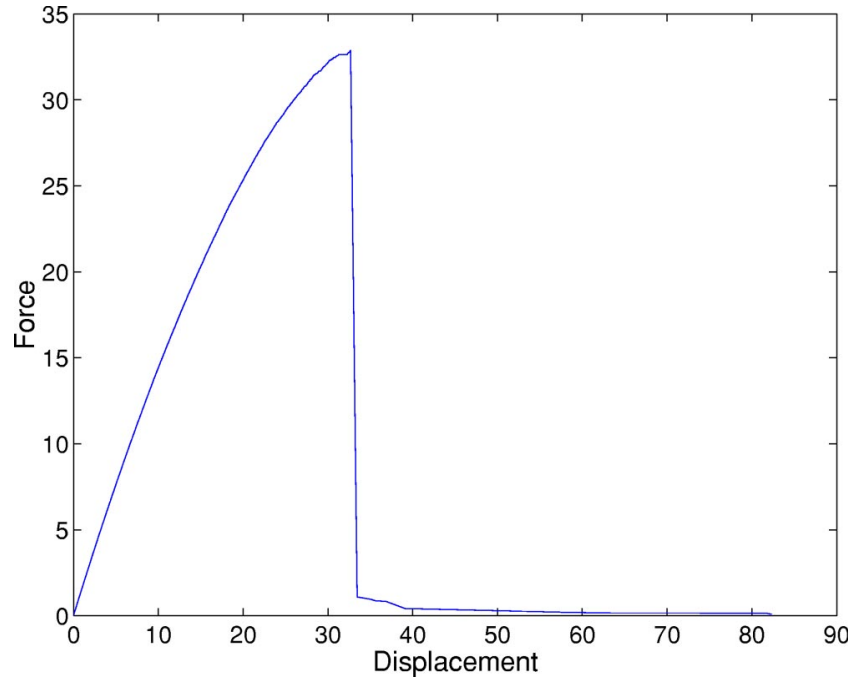

FIG. 1. (Color online) Envelope of a typical force-displacement response obtained using the RSM.

solved to determine the force in each of the springs. Subsequently, for each bond $j$, the ratio between the force $f_{j}$ and the breaking threshold $t_{j}$ is evaluated, and the bond $j_{c}$ having the largest value, $\max _{j}\left(f_{j} / t_{j}\right)$, is irreversibly removed. The
TABLE I. Mean and standard deviation of damage density at the peak load and failure in the random threshold spring model using a triangular lattice network with uniform disorder distribution. $N_{\text {config }}$ denotes the number of configurations used in averaging the results for each system size. $p_{p}$ and $p_{f}$ denote the mean fraction of broken bonds in a lattice system of size $L$ at the peak load and at failure, respectively. Similarly, $\Delta_{p}$ and $\Delta_{f}$ denote the standard deviation of the fraction of broken bonds at the peak load and at failure, respectively.

\begin{tabular}{cccccc}
\hline \hline & \multicolumn{5}{c}{ Triangular } \\
$\mathrm{L}$ & $N_{\text {config }}$ & $p_{p}$ & $\Delta_{p}$ & $p_{f}$ & $\Delta_{f}$ \\
\hline 8 & 40000 & 0.1213 & 0.0285 & 0.2244 & 0.0482 \\
16 & 40000 & 0.1045 & 0.0179 & 0.1869 & 0.0349 \\
24 & 40000 & 0.0970 & 0.0137 & 0.1633 & 0.0258 \\
32 & 40000 & 0.0923 & 0.0113 & 0.1477 & 0.0201 \\
64 & 8000 & 0.0835 & 0.0075 & 0.1175 & 0.0106 \\
128 & 2400 & 0.0763 & 0.0051 & 0.0972 & 0.0056 \\
256 & 100 & 0.0708 & 0.0031 & 0.0836 & 0.0029 \\
\hline \hline
\end{tabular}

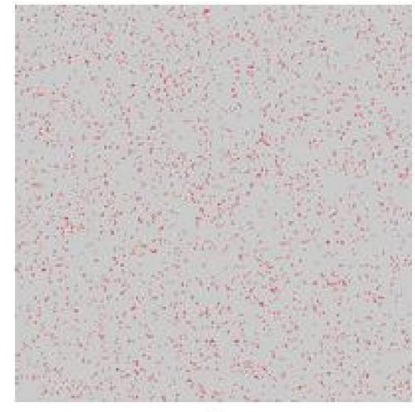

(1)

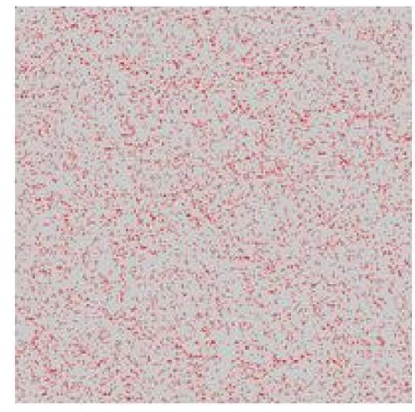

(4)

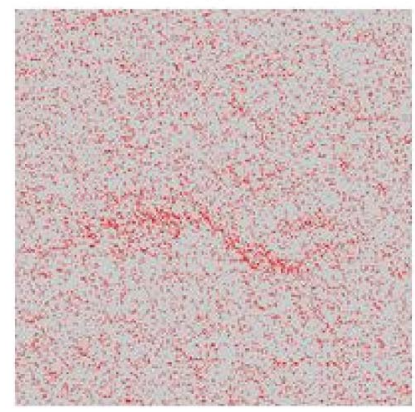

(7)

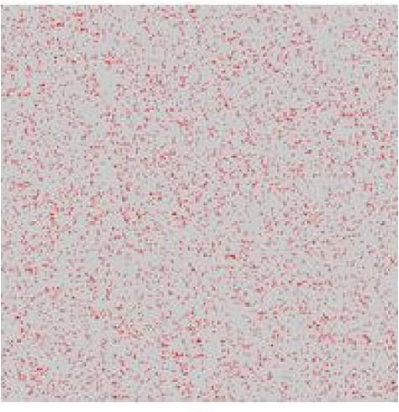

(2)

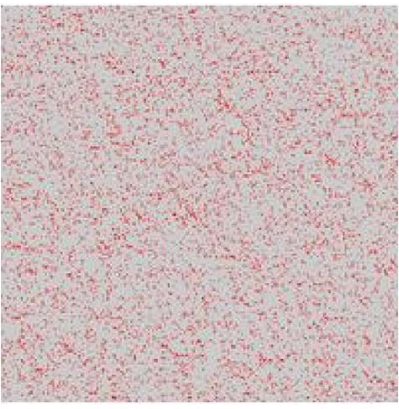

(5)

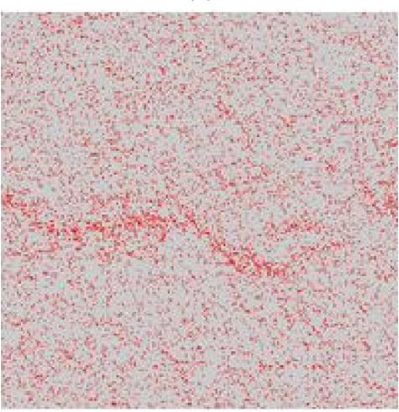

(8)

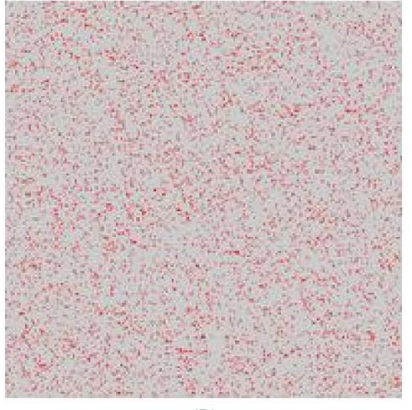

(3)

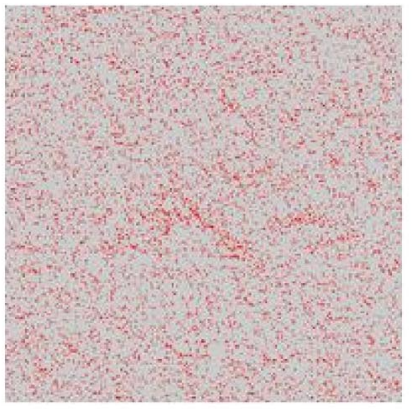

(6)

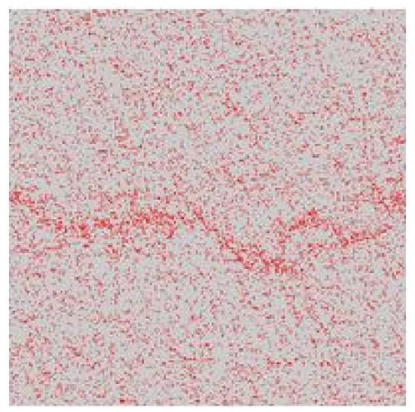

(9)
FIG. 2. (Color online) Snapshots of damage evolution in a typical simulation of size $L=256$. Number of broken bonds at the peak load and at failure is 13864 and 16695 , respectively. (1)-(9) represent the snapshots of damage after $n_{b}$ bonds are broken. (1) $n_{b}$ $=5000 \quad$ (2) $n_{b}=10000$, (3) $n_{b}$ $=12000$, (4) $n_{b}=13000$, (5) $n_{b}$ $=14000$ (just after peak load), (6) $n_{b}=15000$, (7) $n_{b}=15500$, (8) $n_{b}=16000$, and (9) $n_{b}=16500$ (close to failure). 
forces are redistributed instantaneously after a bond is broken, implying that the stress relaxation in the lattice system is much faster than the breaking of a bond. Each time a bond is broken, it is necessary to reequilibrate the lattice system in order to determine the subsequent breaking of a bond. The process of breaking of a bond, one at a time, is repeated until the lattice system falls apart. For the RSM, we consider a triangular lattice system network and a uniform probability distribution for threshold disorder, which is constant between 0 and 1 . The diamond lattice (square lattice with bonds inclined at 45 degrees) spring system exhibits certain unstable modes and hence is not considered. Figure 1 presents the envelope of a typical force-displacement response obtained using the RSM. The peak load of the lattice system is defined as the maximum force of the force-displacement response.

Numerical simulation of fracture using large lattice networks is often hampered due to the high computational cost associated with solving a new large set of linear equations every time a new lattice bond is broken. In this study, we use the multiple-rank sparse Cholesky factorization updating algorithm developed in Ref. [8] for simulating fracture using discrete lattice systems. In comparison with the Fourier accelerated iterative schemes used for modeling lattice breakdown [23], this algorithm significantly reduced the computational time required for solving large lattice systems. For instance, solving for an $L=256$ system takes on average $15300 \mathrm{~s}$ on an IBM power4 $1.3 \mathrm{GHz}$ processor. Using this numerical algorithm, we were able to investigate damage evolution in large ( $L=512$ for spring model) initially fully intact discrete lattice systems. However, due to an insufficient number of available sample configurations, in this paper we consider results up to $L=256$ for spring models. For many lattice system sizes, the number of sample configurations, $N_{\text {config, }}$, used is excessively large to reduce the statistical error in the numerical results (see Table I). In Table I, the fraction of broken bonds (or damage density) for each of the lattice system sizes is obtained by dividing the number of broken bonds with the total number of bonds, $N_{\mathrm{el}}$, present in the fully intact lattice system. For triangular lattice topology, $N_{\mathrm{el}}=(3 L+1)(L+1)$. The lattice system sizes considered in this work are $L=\{8,16,24,32,64,128,256\}$. However, since corrections to the scaling laws are strongest for small lattice systems, in the following we use lattice sizes $L \geqslant 16$ for obtaining the scaling exponents. Table I presents mean and standard deviations in the broken bond density (fraction of broken bonds) at the peak load and at failure for various triangular lattice system sizes.

\section{DIFFUSIVE DAMAGE AND LOCALIZATION}

Qualitatively, damage evolution as described by breaking of bonds is controlled by two competing aspects: disorder and stress concentration in the vicinity of crack tips. In the case of strong disorder, bond-breaking events occur in an uncorrelated manner in the initial stages of damage evolution and thus resemble percolation. As the damage starts to accumulate, some degree of correlation can be expected due to the presence of stress concentration at the crack tips. A natural question to ask concerns the relevance of these correla- tions as failure is approached. If correlations are irrelevant, one should observe percolation scaling up to failure, as in the case of infinite disorder. On the other hand, in the weak disorder case, the current enhancement at the crack tips is so strong that a spanning crack is nucleated soon after a few bonds (or even a single bond) are broken [3]. The interesting situation corresponds to the diffuse damage and localization regime, where a substantial amount of damage is accumulated prior to failure. Figure 2 presents the snapshots of damage evolution in a typical RSM simulation of size $L=256$.

In order to investigate the localization of damage prior to failure, we divided the load-displacement response of a typical RSM simulation into 12 segments, with six equal segments each before and after the peak load. Figures 3 and 4 present the snapshots of damage profiles within each segment of load-displacement curve of a typical simulation with uniform threshold disorder for $L=256$. The damage is diffusive in the initial stages of loading up to almost the peak load. Around the peak load, the damage starts to localize and ultimately leads to failure, and hence the final breakdown event is very different from the initial precursors up to the peak load. Based on Figs. 3 and 4, it is clear that localization of damage occurs in the RSM prior to failure even for strong but finite disorder. Similar behavior is observed in the random threshold fuse model with both uniform and power-law threshold distributions [22].

In order to obtain a quantitative description of the damage localization process, it is necessary to average the damage profiles over different realizations. Since the localization of damage can occur anywhere along the $y$ direction of the lattice, a simple averaging of the damage profiles would yield a flat profile irrespective of the individual profile shapes in a single realization. In this study, we average the damage profiles by first shifting the damage profiles by the center of mass of the damage and then averaging. Alternatively, one could average the magnitude of the Fourier transforms of individual damage profiles, thereby retaining the frequency content of damage profiles. The Fourier method eliminates any artificial biasing associated with the shifting of the individual profiles in the real space [22].

Figure 5 presents the average damage profiles for the damage accumulated up to the peak load by first shifting the damage profiles by the center of mass of the damage and then averaging over different samples. The results presented in Fig. 5 indicate that although the average damage profiles at smaller lattice system sizes are not completely flat, they flatten considerably as the lattice system size is increased. We tend thus to attribute the apparent profile to size effects. Indeed, for large system sizes (e.g., $L=128$ and 256), the results clearly show that there is no localization at the peak load. Consequently, the localization of damage is mostly due to the damage accumulated between the peak load and failure, i.e., the final catastrophic breakdown event. Figure 6 presents the data collapse of the average damage profiles for the damage accumulated between the peak load and failure using a power-law scaling. A perfect collapse of the data is obtained using the form

$$
\langle\Delta p(y, L)\rangle /\langle\Delta p(0)\rangle=f(|y-L / 2| / \xi),
$$

where the damage peak scales as $\langle\Delta p(0)\rangle=L^{-0.37}$ and the localization length scales as $\xi \sim L^{\alpha}$, with $\alpha=0.65$ (see Fig. 6). 

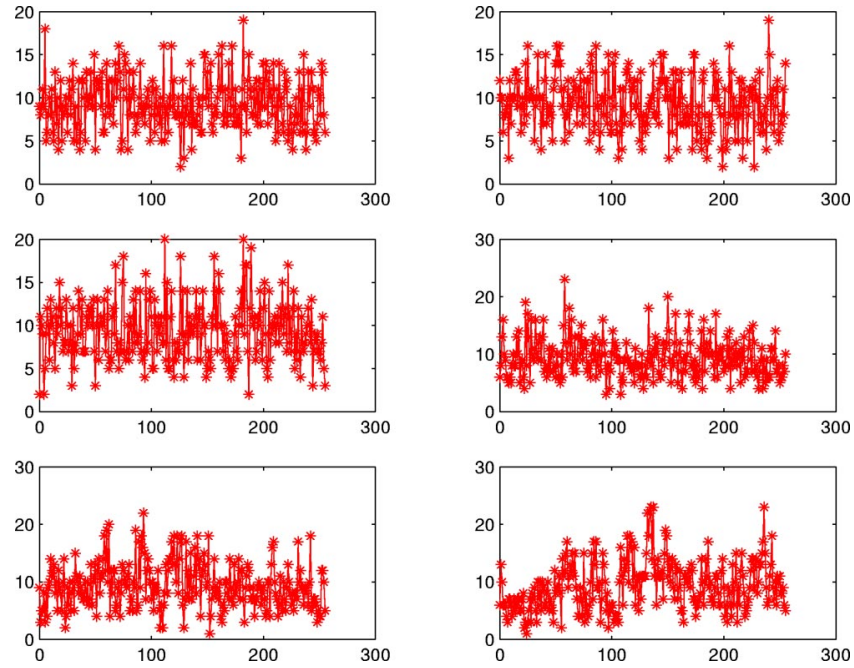

FIG. 3. (Color online) Snapshots of prepeak damage profiles of a typical RSM simulation with uniform threshold distribution on a triangular lattice of size $L=256$. The damage is uniform in the prepeak regime. In each of the subplots, the abscissa refers to the $y$ coordinate of the lattice section and the ordinate is the number of broken bonds in the section.

The profile shapes decay exponentially at large system sizes. We have also tried a simple linear scaling of the form $\langle\Delta p(y, L)\rangle /\langle\Delta p(0)\rangle=f[(y-L / 2) / L]$, but the collapse of the data is not very good. The result for the fuse model is similar: the profile also displays exponential tails and the exponent is found to be $\alpha=0.8$ [22] .

\section{SCALING OF DAMAGE DENSITY}

It has been noted in the previous section that the final breakdown event is very different from the initial precursors.
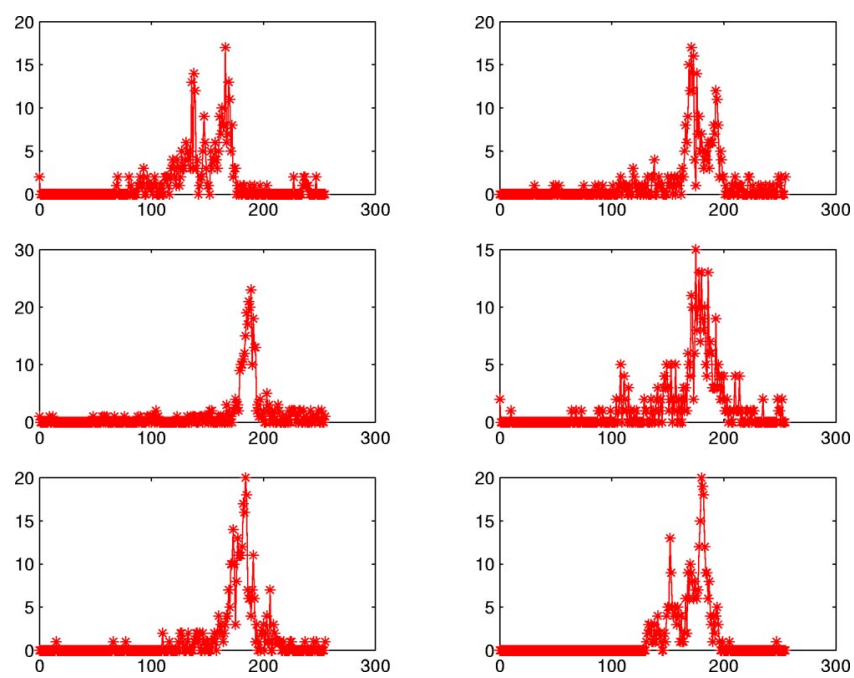

FIG. 4. (Color online) Snapshots of post-peak damage profiles of a typical RSM simulation with uniform threshold distribution on a triangular lattice of size $L=256$. The damage is clearly localized in the post-peak regime. In each of the subplots, the abscissa refers to the $y$ coordinate of the lattice section and the ordinate is the number of broken bonds in the section.

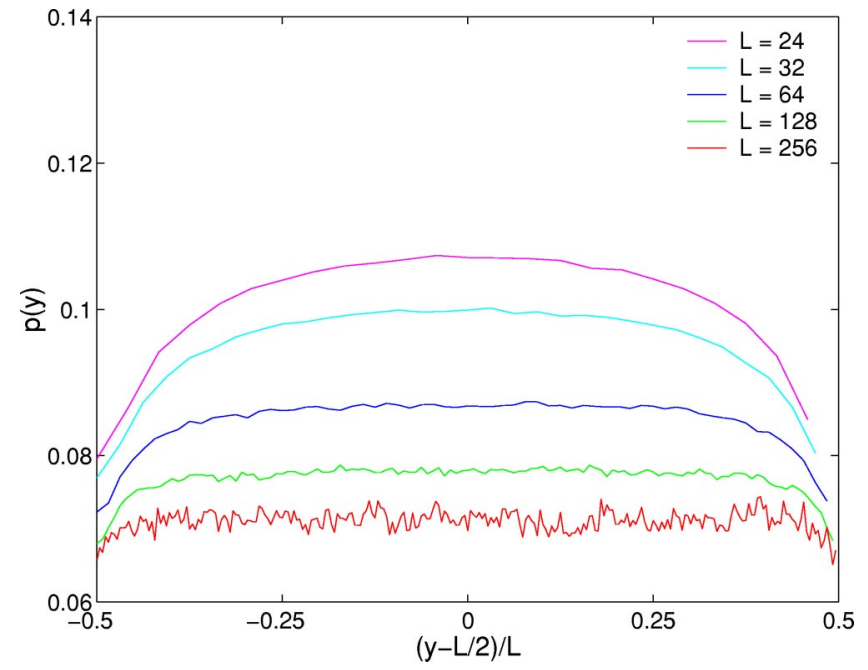

FIG. 5. (Color online) Average damage profiles at peak load obtained by first centering the data around the center of mass of the damage and then averaging over different samples. For each of the samples, the damage profile is evaluated as $p(y)=n_{b}(y) /(3 L+1)$, where $n_{b}(y)$ denotes the number of broken bonds in the $y$ th section.

Thus, we consider the scaling of the number of broken bonds at the peak load, $n_{p}$, that excludes the last catastrophic event. In Fig. 7, we plot $n_{p}$ as a function of the lattice size $N_{\mathrm{el}}$. The data display a reasonable power-law behavior $n_{p} \sim N_{\mathrm{el}}^{b}$, with $b=0.92$. The exponent $b=0.92$ is in close agreement with the value obtained for the random threshold fuse model using both triangular $(b=0.93)$ and diamond $(b=0.91)$ lattice topologies [22]. The difference between the RSM and RFM models is marginal and may be attributed to the results obtained from the smaller lattice sizes, where corrections to the fractal scaling may exist. However, we have noticed some

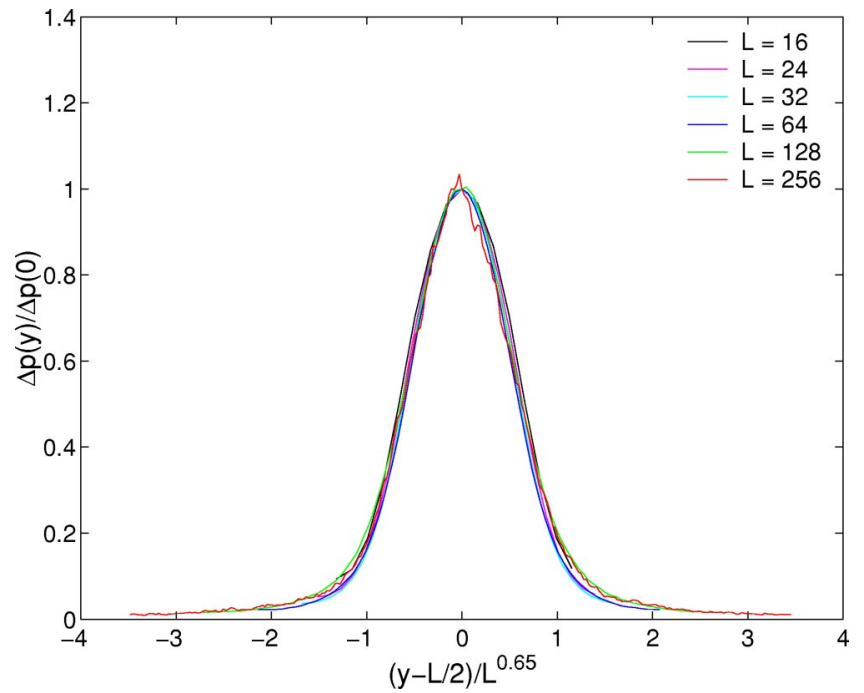

FIG. 6. (Color online) Data collapse of the average profiles for the damage accumulated between peak load and failure using a power-law scaling. We have considered the damage profiles for $L$ $=\{16,24,32,64,128,256\}$ system sizes. The average has been performed after shifting by the center of mass. The profiles show exponential tails. 


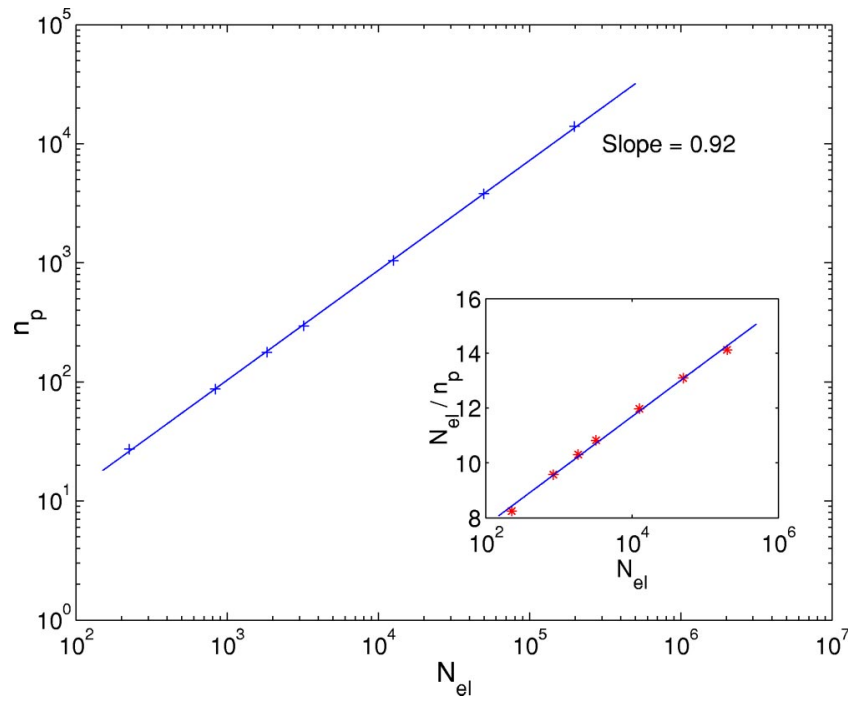

FIG. 7. (Color online) Scaling of the number of broken bonds at peak load for triangular random threshold spring lattices. The scaling exponent $b=0.92$ is very close to the exponent obtained for a random threshold fuse network using triangular $(b=0.93)$ and diamond $(b=0.91)$ lattices. The difference could be attributed to finitesize effects. The number of broken bonds at peak load can also be fit by a linear function times a logarithmic correction by plotting $n_{p} / N_{\mathrm{el}}$ as a function of $N_{\mathrm{el}}$ in a log-linear plot (inset).

systematic deviations from the scaling form $n_{p} \sim N_{\mathrm{el}}^{b}$ by plotting $n_{p} / N_{\mathrm{el}}^{b}$ versus $N_{\mathrm{el}}$. Since the exponent $b$ is close to 1 , the data could be equally well fit by a linear law times a logarithmic correction $n_{p} \simeq N_{\mathrm{el}} / \log \left(N_{\mathrm{el}}\right)$, as suggested in Ref. [24] (see the inset of Fig. 7). Both of these fits imply that in the limit of large lattices, the fraction of broken bonds prior to fracture vanishes.

\section{SCALING OF DAMAGE DENSITY PROBABILITY DISTRIBUTIONS}

Since the final breakdown event is very different from the initial precursors up to the peak load, in this section we present the scaling of the cumulative probability distributions for the fraction of broken bonds at the peak load. The cumulative probability distribution for the damage density at the peak load is defined as the probability $\Pi_{p}\left(p_{b}, L\right)$ that a system of size $L$ reaches peak load when the fraction of broken bonds equals $p_{b}=n_{b} / N_{\mathrm{el}}$, where $n_{b}$ is the number of broken bonds. Figure 8 presents the cumulative probability distribution for the damage density at the peak load in the random threshold spring model for various system sizes $L$. By simply plotting the distribution in terms of $\bar{p}_{p} \equiv\left(n_{b}-\mu_{n_{p}}\right) / \sigma_{n_{p}}=\left(p_{b}\right.$ $\left.-p_{p}\right) / \Delta_{p}$, where $\mu_{n_{p}}$ and $\sigma_{n_{p}}$ denote the mean and standard deviation of the number of broken bonds at peak load, and $p_{p}$ and $\Delta_{p}$ denote the mean and standard deviation of fraction of broken bonds at peak load (see Table I), we obtain a very good collapse of the cumulative probability distribution of the damage density at the peak load. Figure 9 shows that $\Pi_{p}(p, L)$ may be expressed in a universal scaling form such that $\Pi_{p}(p, L)=\Pi_{p}\left(\bar{p}_{p}\right)$ for different system sizes $L$. A similar

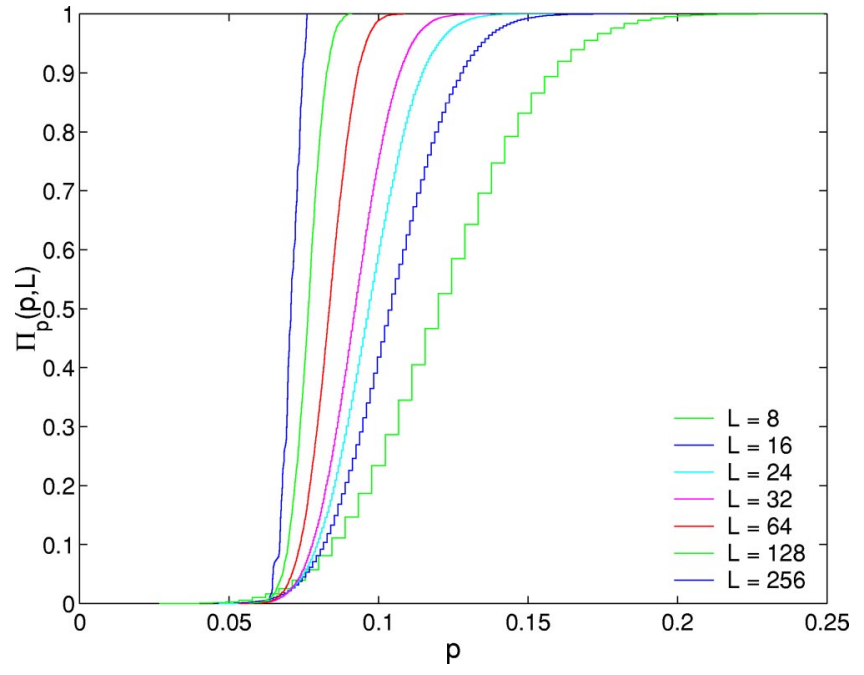

FIG. 8. (Color online) The cumulative probability distribution for the fraction of broken bonds at the peak load for triangular spring lattices of different system sizes.

collapse has been performed for the random threshold fuse model in Ref. [22]. The inset in Fig. 9 presents a comparison of the cumulative damage density probability distributions in the random threshold spring and fuse models. The excellent collapse of the data in the inset of Fig. 9 suggests that the

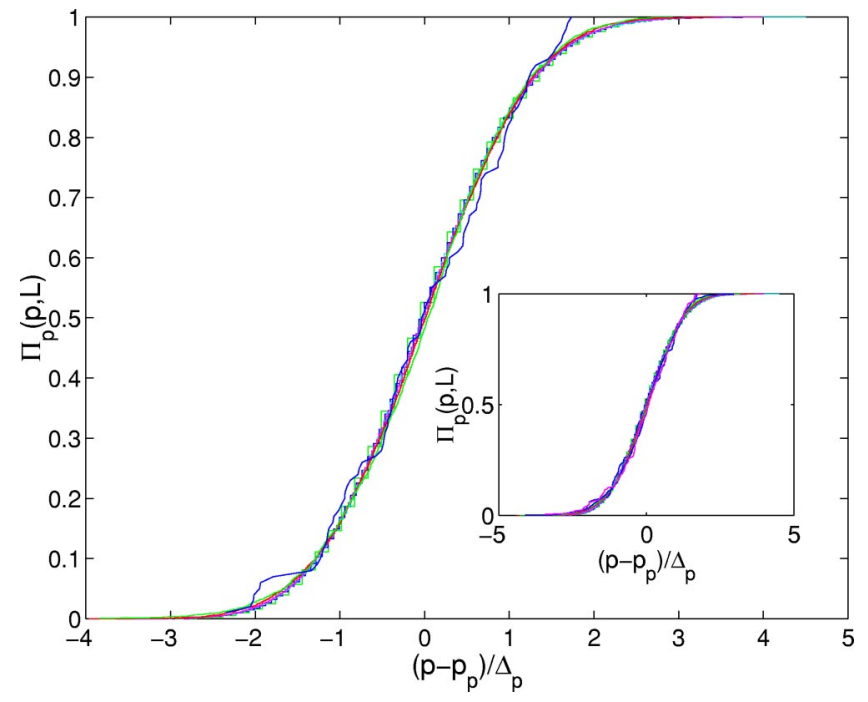

FIG. 9. (Color online) The collapsed cumulative probability distribution for the fraction of broken bonds at the peak load in the random threshold spring model (RSM) using triangular lattices of different system sizes $(L=8,16,24,32,64,128,256)$ with uniform disorder when plotted as a function of the reduced variable $\bar{p}_{p}=(p$ $\left.-p_{p}\right) / \Delta_{p}$. In the inset, a comparison between the cumulative probability distributions of the fraction of broken bonds at the peak load is presented for the RSM and RFM. For the RSM, triangular lattices of sizes $(L=8,16,24,32,64,128,256)$ and for the RFM, triangular lattices of sizes $(L=16,24,32,64,128,256,512)$ are plotted. In the RFM case, the collapse of cumulative probability distributions at the peak load for different lattice topologies (triangular and diamond) and different disorder distributions (uniform and power law) is presented in Ref. [22]. 


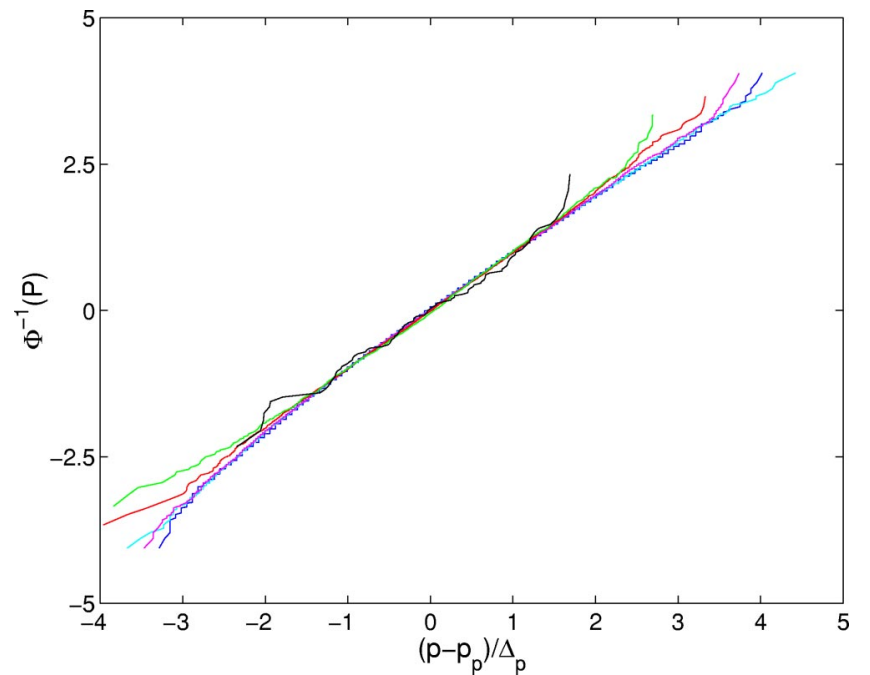

FIG. 10. (Color online) Normal distribution fit for the cumulative probability distributions of the fraction of broken bonds at the peak load for triangular spring lattices of different system sizes $L$ $=\{8,16,24,32,64,128,256\}$.

cumulative probability distribution for the damage density at the peak load, $\Pi_{p}\left(p_{b}, L\right)=\Pi_{p}\left(\bar{p}_{p}\right)$, may be universal. Finally, the collapse of the data in Fig. 10 indicates that a Gaussian distribution adequately describes $\Pi_{p}$.

In Ref. [22], we have also checked that the distributions at failure in the random threshold fuse model obey essentially the same laws, i.e., $\Pi(\bar{p})=\Pi_{f}\left(\bar{p}_{f}\right)=\Pi_{p}\left(\bar{p}_{p}\right)$, where $\Pi_{f}\left(\bar{p}_{f}\right)$ is the probability that a system of size $L$ fails when the fraction of broken bonds equals $p_{b}$, and $\bar{p}_{f}$ is the corresponding reduced variable at failure. However, in the RSM, although a reasonable collapse of the cumulative probability distribution of damage density at failure can be obtained, the cumulative distributions of damage density at peak load and at failure appear to be different. In particular, the distribution, $\Pi_{f}\left(\bar{p}_{f}\right)$, at failure is not adequately described by a Gaussian distribution. The inadequacy of a Gaussian distribution in the post- peak regime may indicate the presence of a relatively stronger localization in the RSM compared with the RFM.

\section{UNIVERSALITY OF FRACTURE STRENGTH DISTRIBUTION}

In this study, we start the numerical simulation with a fully intact lattice system. The fracture strength of such a system is defined as the stress corresponding to the peak load of the lattice system response.

Figure 11(a) presents the fracture strength density distributions for a random threshold spring model using the standard $\log$-normal variable, $\bar{\xi}$, defined as $\bar{\xi}=\left[\ln \left(\sigma_{f}\right)-\eta\right] / \zeta$, where $\sigma_{f}$ refers to the fracture strength defined as the peak load divided by the system size $L$, and $\eta$ and $\zeta$ refer to the mean and the standard deviation of the logarithm of $\sigma_{f}$. In order to verify the universality of fracture strength distribution, the fracture strength distributions from [17] corresponding to the random threshold fuse model (RFM) using triangular lattice systems with uniform disorder are presented in Fig. 11(a) along with those corresponding to the random threshold spring model. In particular, Fig. 11(a) shows the data for different lattice system sizes, $L$, corresponding to (a) triangular spring lattice, $L=\{8,16,24,32,64,128\}$ and (b) triangular fuse lattices of sizes $L=\{4,8,16,24,32,64,128\}$. In all, there are 13 plots in Fig. 11(a), and the excellent collapse of the data for various spring and fuse lattices clearly indicates the universality of the fracture strength density distribution. The results presented in Fig. 11(a) are limited only up to a system size of $L=128$ due to the availability of fewer sample configurations for larger lattice systems. In order to attain a good collapse of the data for the density distributions, it is necessary to consider many sample configurations. On the other hand, good collapse of the data for the cumulative distributions can be achieved using fewer sample configurations. Figure 11(b) presents the cumulative fracture strength versus the standard log-normal variable, $\bar{\xi}$, for random spring and fuse lattice networks for system sizes
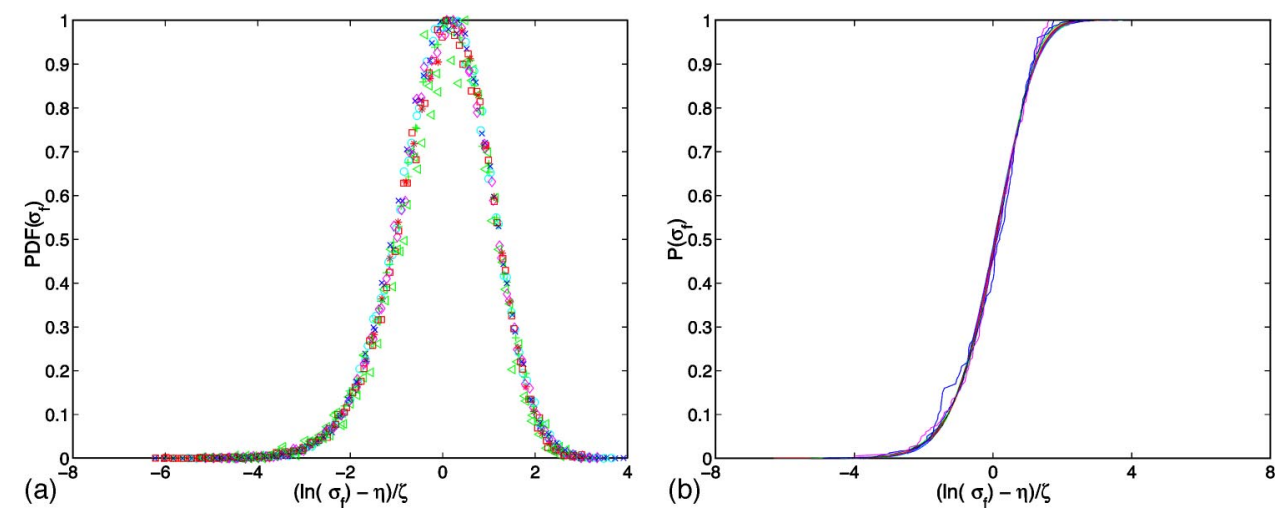

FIG. 11. (Color online) Universality of fracture strength distribution in the random threshold spring and fuse models. (a) Fracture strength density distributions for triangular spring lattices $(L=\{8,16,24,32,64,128\})$ and triangular fuse lattices $(L=\{4,8,16,24,32,64,128\})$ with uniform disorder. (b) Cumulative fracture strength distribution for triangular spring lattices $(L=\{8,16,24,32,64,128,256\})$ and triangular fuse lattices $(L=\{4,8,16,24,32,64,128,256,512\})$ with uniform disorder. The collapse of the data in random spring and fuse models suggests universality of fracture strength distribution. In the RFM case, the universality of fracture strength distributions with respect to different lattice topologies is presented in Ref. [17]. 

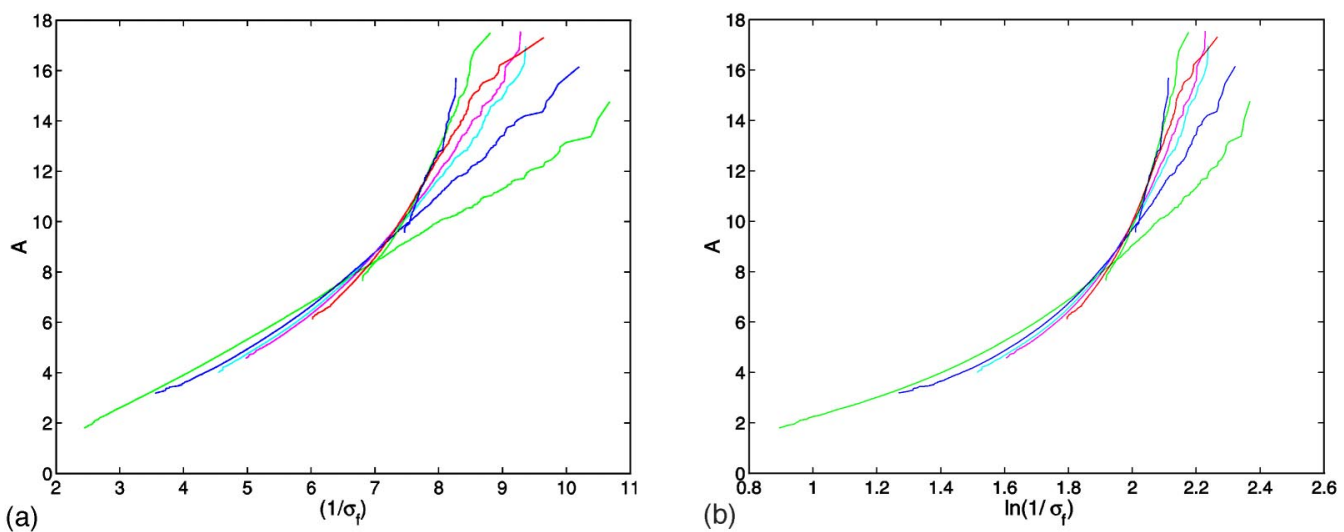

(a)

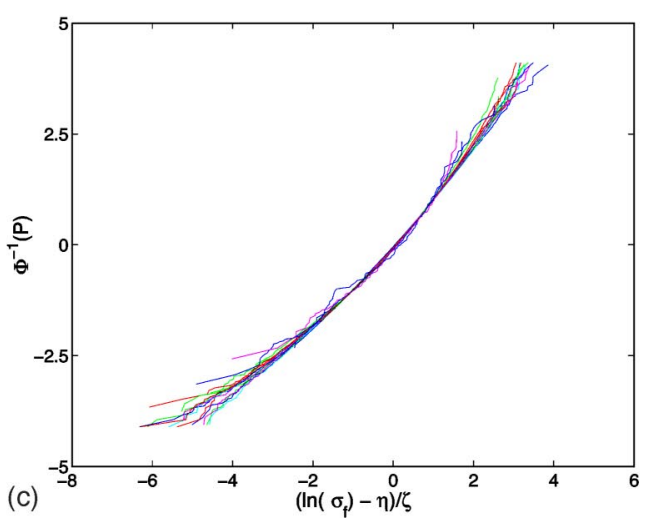

FIG. 12. (Color online) Probability distribution fits for fracture strengths at the peak load in a triangular spring lattice network for different lattice system sizes $L=\{8,16,24,32,64,128,256\}$. (a) Modified Gumbel distribution (top). (b) Weibull distribution (middle). (c) Log-normal distribution fit for all the 16 curves [see Fig. 11(b)] (bottom). Since the data for different lattice system sizes do not collapse onto a single curve, Weibull distribution may not be an adequate fit for representing fracture strengths in the RSM. On the other hand, the collapse of the data in the reparametrized log-normal distribution fit suggests that the log-normal distribution describes the fracture strength distribution adequately.

up to $L=512$. In particular, in Fig. 11(b), we plot the numerical simulation results of RSM for system sizes $L$ $=\{8,16,24,32,64,128,256\}$ along with those of RFM for system sizes $L=\{4,8,16,24,32,64,128,256,512\}$. In all, there are about 16 curves ( 7 for triangular spring lattices and 9 for triangular fuse lattices) in Fig. 11(b), and the excellent collapse of the data suggests universality of fracture strength distribution. In Ref. [17], we have also presented the collapse of the fracture strength distribution for different lattice topologies (such as triangular and diamond), which is consistent with the notion of universality of fracture strength distribution. That is, $P\left(\sigma \leqslant \sigma_{f}\right)=\Psi(\bar{\xi})$, where $P\left(\sigma \leqslant \sigma_{f}\right)$ refers to the cumulative probability of fracture strength $\sigma \leqslant \sigma_{f}, \Psi$ is a universal function such that $0 \leqslant \Psi \leqslant 1$, and $\bar{\xi}=\left[\ln \left(\sigma_{f}\right)\right.$ $-\eta] / \zeta$ is the standard log-normal variable.

Figures 12(a) and 12(b) present the modified Gumbel and Weibull fits for the fracture strength distribution of a triangular spring lattice network using

$$
A=k\left(\frac{1}{\sigma_{f}^{\delta}}\right)-\ln c
$$

for the modified Gumbel distribution, and

$$
A=m \ln \left(\frac{1}{\sigma_{f}}\right)-\ln c
$$

for the Weibull distribution. In Eqs. (2) and (3), $k, \delta, c$, and $m$ are constants, and $A$ is defined as

$$
A=-\ln \left[-\frac{\ln \left[1-P\left(\sigma_{f}\right)\right]}{L^{2}}\right],
$$

where $P\left(\sigma_{f}\right)$ denotes the cumulative distribution. From these figures, it is clear that fracture strength data for different lattice system sizes do not collapse onto a single straight line as they should, if the data were to follow Eq. (2) or Eq. (3). This indicates that neither modified Gumbel nor Weibull distributions may represent the fracture strengths distribution accurately for the RSM. In Ref. [17], similar conclusion has been drawn for the fracture strengths distribution of RFM.

On the other hand, in Fig. 12(c), we test the log-normal description for fracture strengths by plotting the inverse of the cumulative probability, $\Phi^{-1}\left[P\left(\sigma_{f}\right)\right]$, against the standard log-normal variable, $\bar{\xi}$. In the above description, $\Phi(\cdot)$ denotes the standard normal probability function. In particular, in Fig. 12(c) we present the log-normal fit for the cumulative fracture strength distributions obtained for random threshold spring and fuse models [i.e., for all of the 16 curves in Fig. 
TABLE II. Peak load in the random threshold spring model using a triangular lattice network with uniform disorder distribution. $N_{\text {config }}$ denotes the number of configurations used in averaging the results for each system size.

\begin{tabular}{cccc}
\hline \hline & & \multicolumn{2}{c}{ Triangular } \\
$\mathrm{L}$ & $N_{\text {config }}$ & Mean & Std \\
\hline 8 & 40000 & 1.8125 & 0.3318 \\
16 & 40000 & 2.8646 & 0.3364 \\
24 & 40000 & 3.9170 & 0.3558 \\
32 & 40000 & 4.9619 & 0.3761 \\
64 & 8000 & 9.0865 & 0.4632 \\
128 & 2400 & 17.1286 & 0.6122 \\
256 & 100 & 32.8959 & 0.8024 \\
\hline \hline
\end{tabular}

11(b)]. Once again, this figure clearly indicates that the fracture strength distribution obtained for different lattice system sizes collapses onto a single curve, although a minute deviation from straight line behavior is evident. The log-normal distribution can be understood to have evolved as a consequence of the multiplicative nature of a large number of random distributions representing the stress scale factors necessary to break the subsequent "primary" bonds (by definition, an increase in applied stress is required to break a "primary" bond) leading up to the peak load [17]. As long as the number of "primary" broken bonds is large, the fracture strength probability distribution approaches a log-normal distribution (by central limit theorem) irrespective of the precise character of the individual stress scale factor random distributions [17]. We have also used the normal distribution to collapse the fracture strength data of triangular spring and fuse lattice systems. Although the data collapse is reasonable, it is not as good as that of the log-normal distribution based on the Kolmogorov-Smirnov goodness-of-fit test.

\section{SIZE EFFECTS IN THE MEAN FRACTURE STRENGTH}

The mean fracture strength data for various random threshold spring lattice system sizes are presented in Table II. In Ref. [17], for the RFM, we have suggested a scaling form $\bar{F}_{\text {peak }}=C_{0} L^{\bar{\alpha}}+C_{1}$ for the peak load, where $C_{0}$ and $C_{1}$ are constants. Correspondingly, the mean fracture strength defined as $\mu_{f}=\bar{F}_{\text {peak }} / L$, is given by $\mu_{f}=C_{0} L^{\bar{\alpha}-1}+\left(C_{1} / L\right)$. We have used the same scaling law for the random threshold spring model as well, and the result presented in Fig. 13 indicates that the exponent $\bar{\alpha}$ is approximately equal to 0.97 , which is once again consistent with the $\bar{\alpha}=0.96$ obtained for RFM using both triangular and diamond lattice topologies. The inset in Fig. 13 presents a power-law fit $\mu_{f} \sim L^{-2 / m}$ that is consistent with a Weibull distribution for fracture strengths. From the nonlinearity of the plots in the inset of Fig. 13, it is clear that the mean fracture strength does not follow a simple power-law scaling that is consistent with a Weibull distribution for fracture strengths. We have also verified that the mean fracture strength does not follow a scaling law of the

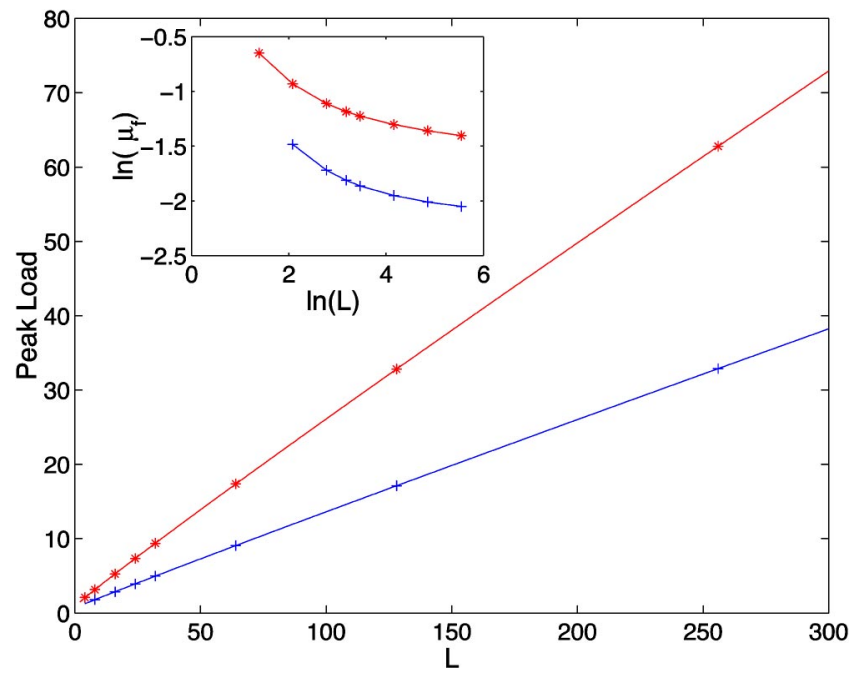

FIG. 13. (Color online) Proposed scaling law for the mean fracture strength $\left(F_{\text {peak }}=C_{0} L^{\bar{\alpha}}+C_{1}\right)$. (1) Triangular spring network (symbol: -+-): $\bar{\alpha}=0.97$. (2) Triangular fuse network (symbol: $-^{*}-$ ): $\bar{\alpha}=0.956$; the corresponding Weibull fit for the mean fracture strength is shown in the inset. Nonlinearity of the plots in the inset suggests that mean fracture strength does not follow a power-law scaling consistent with the Weibull distribution.

form $\mu_{f}^{\delta}=1 /\left(A_{1}+B_{1} \ln L\right)$ that is consistent with a modified Gumbel distribution for fracture strengths [13-16].

Since a very small negative exponent $(\bar{\alpha}-1)$ is equivalent to a logarithmic correction, i.e., for $(1-\bar{\alpha}) \ll 1, L^{\bar{\alpha}-1}$ $\sim[\log (L)]^{-\psi}$, an alternative expression for the mean fracture strength may be obtained as $\mu_{f}=\mu_{f}^{\star} /(\log L)^{\psi}+(c / L)$, where $\mu_{f}^{\star}$ and $c$ are constants that are related to the constants $C_{0}$ and $C_{1}$. This suggests that the mean fracture strength of the lattice system decreases very slowly with increasing lattice system size, and scales as $\mu_{f} \approx 1 /(\log L)^{\psi}$, with $\psi \approx 0.15$, for very large lattice systems.

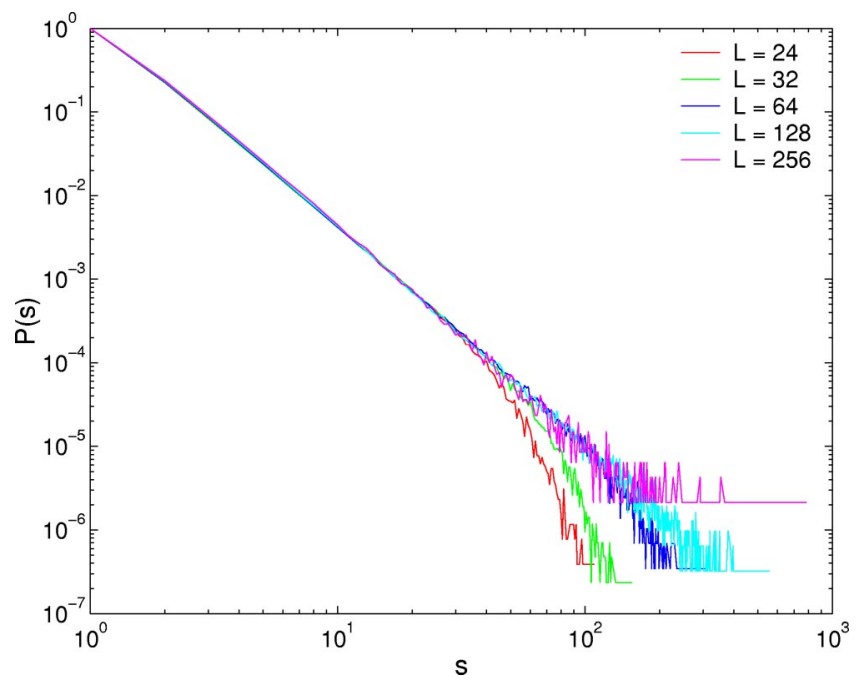

FIG. 14. (Color online) The distribution of avalanche sizes (without the last catastrophic event) for triangular spring lattices of different sizes. 


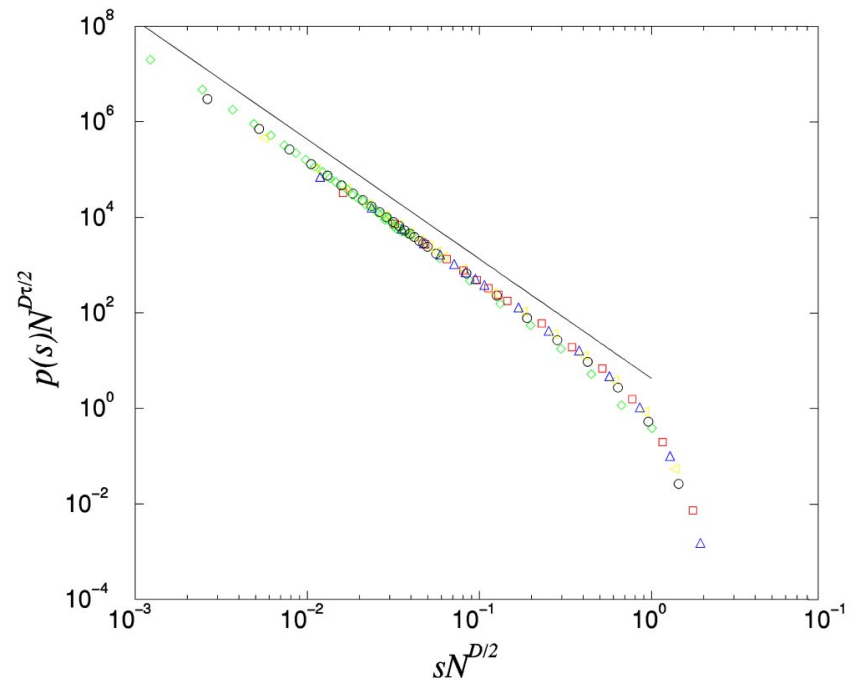

FIG. 15. (Color online) Data collapse of the avalanche size distributions excluding the final catastrophic event. The exponents used for the collapse are $\tau=2.5$ (the reference line has this slope) and $D=1.1$. The distributions have been logarithmically binned to reduce fluctuations.

\section{AVALANCHES}

The avalanche size distribution, once the last event is excluded, is a power law followed by an exponential cutoff at large avalanche sizes (see Fig. 14) [25]. The cutoff size $s_{0}$ increases with the lattice size, so that we can describe the distribution by a scaling form

$$
P(s, N)=s^{-\tau} g\left(s / N^{D / 2}\right),
$$

where $D$ represents the fractal dimension of the avalanches and $N=(3 L+1)(L+1)$ is the number of bonds. Figure 15 pre-

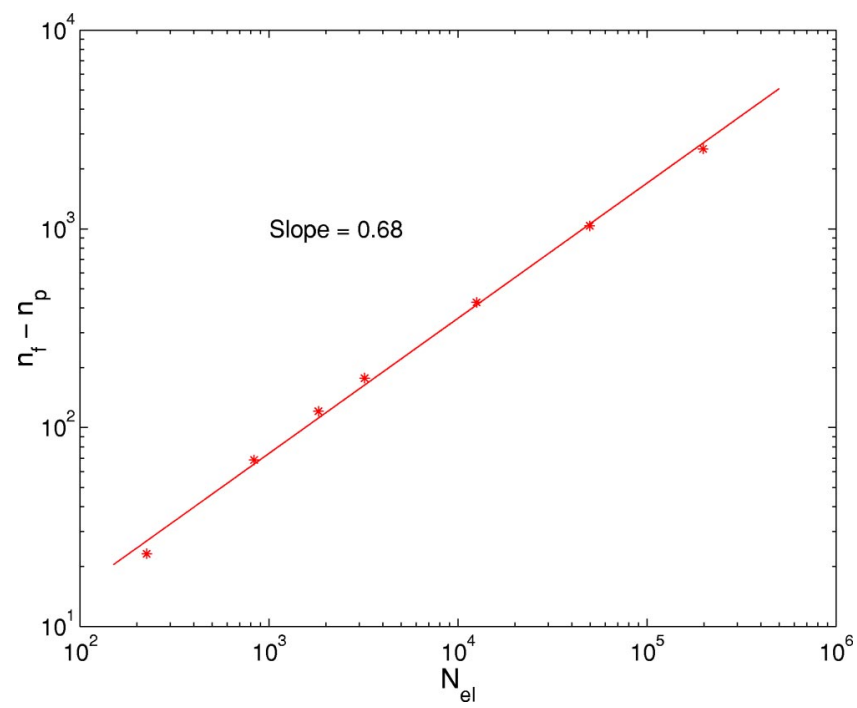

FIG. 16. (Color online) The mean avalanche size of the last catastrophic event $\left[s_{m}=\left(n_{f}-n_{p}\right)\right]$ scales as a power law of $N_{\mathrm{el}}$. Once again, the scaling exponent $b=0.68$ for RSM is similar to the scaling exponent $b \simeq 0.7$ obtained for RFM using triangular and diamond lattices (see Fig. 14 of Ref. [22]).
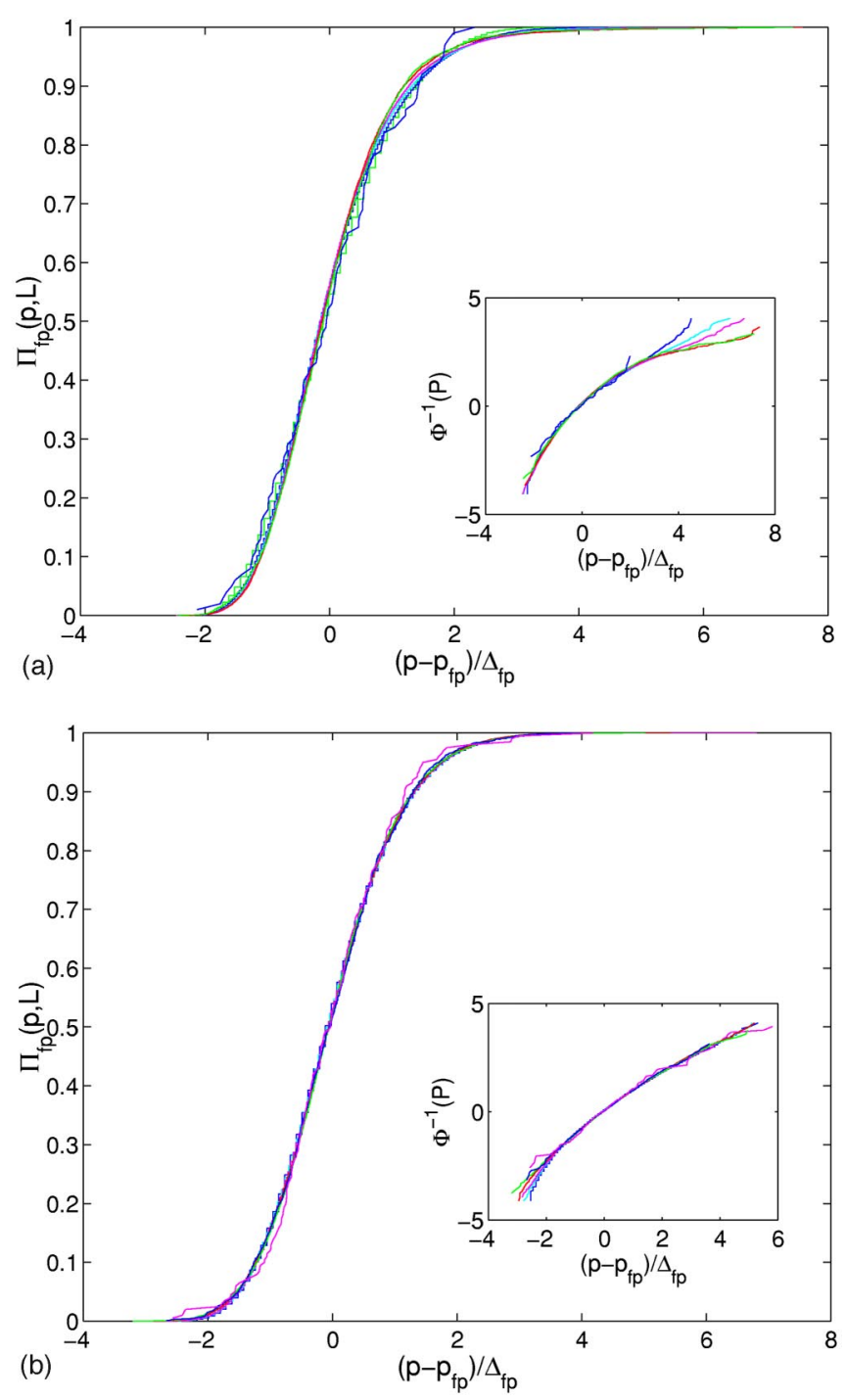

FIG. 17. (Color online) The collapsed cumulative distribution of the last avalanche. (a) RSM, (b) RFM. The insets in each of these figures show how well the data can be represented by a normal distribution fit. The presence of significant nonlinearity of the data in these insets suggests that normal distribution may not be an adequate fit for representing the distribution of the last avalanche size for the RSM model, whereas it may be an adequate fit for the RFM model.

sents the data collapse of the distribution of avalanche sizes using the exponents $\tau=2.5$ and $D=1.1$.

So far we have considered avalanche statistics integrating the distribution over all the values of the forces up to the peak load, but the avalanche signal is not stationary: as the force increases, so does the avalanche size. In particular, the last avalanche is much larger than the others. Its typical size grows as $s_{m}=\left(n_{f}-n_{p}\right) \sim N^{b}$, with $b \simeq 0.68$ (see Fig. 16), which is once again in good agreement with the $b \simeq 0.7$ value obtained for RFM (see Fig. 14 of Ref. [22]). The cumulative distribution of the last avalanche sizes for the RSM and RFM is presented in Figs. 17(a) and 17(b), respectively. While the distribution is approximately Gaussian for RFM as shown by the data collapse (almost linear) in the inset of Fig. 17(b), 
there appears to be significant nonlinearity in the data collapse of the plots in the inset of Fig. 17(a). This suggests that normal distribution may not be an adequate fit for representing the distribution of the last avalanche size in the RSM model. We notice here that the postpeak regime is different in the two models because the RSM can fail due to loss of rigidity. In general, the significantly different nature of the last avalanche with respect to the precursors is revealed both by the distribution type (Gaussian or power law) and by its characteristic value, scaling as $2 b \simeq 1.36$ or $D=1.1$. This difference reflects the fact that the last avalanche is a catastrophic event corresponding to unstable crack growth, while precursors reflect metastable crack growth and the two processes are different.

\section{SUMMARY}

In this study, we investigated the universality of random threshold spring and fuse models using large-scale numerical simulations and a large number of sample configurations to reduce the statistical error in the numerical results. For both models, we considered triangular lattice topology with uniform disorder and increased the load quasistatically. We performed several statistical measures characterizing the fracture process that can be summarized as follows.

(i) Damage localization. The process of localization is similar in the RFM and RSM. Damage is accumulated in a uniform manner up to the peak load and then suddenly localizes, leading to complete failure. This process is described by damage profiles that are basically flat until peak load and show a peak, with exponential tails, in the postpeak regime. The collapse of the damage profiles implies some small differences in the exponents for the two models.

(ii) Damage density. The number of broken bonds at failure or at peak load scales with the lattice size. We have shown that the behavior in RFM and RSM is very similar, but in both cases it is not possible to distinguish a power law from a linear behavior with a logarithmic correction.

(iii) Damage distributions. The distribution of broken bonds at peak load follows a normal distribution for RFM and RSM.

(iv) Fracture strength. The fracture strength distribution is found to be log-normal for both models and the mean fracture strength scales logarithmically.

(v) Avalanches. The integrated avalanche distributions follow a power law in both models. The results of the RSM yield an exponent $\tau=5 / 2$ that is very close to the exponent observed in the global load sharing fiber bundle model (FBM), while larger deviations are found in the RFM (i.e., $\tau=2.7[12])$.

Thus, in conclusion, we can state that RFM and RSM are qualitatively very similar: distributions have the same forms, localization proceeds in the same way, and avalanches are similar. However, minor differences can be found in terms of small quantitative deviations in the exponents. We cannot rule out that these deviations are due to differences in the finite-size behavior of the models and that at large scales the behavior is the same. In addition, the rigidity mechanism that is present in the RSM and not in the RFM could explain some deviations in the postpeak regime. Our analysis suggests that a simplified scalar model can capture all the essential features of a numerically more expensive tensorial model. Hence, the RFM could be considered as the basic model to investigate the statistical properties of fracture in disordered media.

\section{ACKNOWLEDGMENTS}

This research is sponsored by the Mathematical, Information and Computational Sciences Division, Office of Advanced Scientific Computing Research, U.S. Department of Energy under Contract No. DE-AC05-00OR22725 with UTBattelle, LLC.
[1] Statistical Models for the Fracture of Disordered Media, edited by H. J. Herrmann and S. Roux (North-Holland, Amsterdam, 1990); B. K. Chakrabarti and L. G. Benguigui, Statistical Physics of Fracture and Breakdown in Disordered Systems (Oxford University Press, Oxford, 1997).

[2] L. de Arcangelis, S. Redner, and H. J. Herrmann, J. Phys. (Paris), Lett. 46, 585 (1985).

[3] B. Kahng, G. G. Batrouni, S. Redner, L. de Arcangelis, and H. J. Herrmann, Phys. Rev. B 37, 7625 (1988).

[4] L. de Arcangelis, A. Hansen, H. J. Herrmann, and S. Roux, Phys. Rev. B 40, 877 (1989).

[5] A. Delaplace, G. Pijaudier-Cabot, and S. Roux, J. Mech. Phys. Solids, 44, 99 (1996).

[6] S. Zapperi, P. Ray, H. E. Stanley, and A. Vespignani, Phys. Rev. Lett. 78, 1408 (1997); Phys. Rev. E 59, 5049 (1999).

[7] A. Hansen and S. Roux, Statistical Toolbox for Damage and Fracture, in Damage and Fracture of Disordered Materials, edited by D. Krajcinovic and van Mier (Springer Verlag, New
York, 2000), pp. 17-101.

[8] P. K. V. V. Nukala and S. Simunovic, J. Phys. A 36, 11403 (2003).

[9] S. Roux, A. Hansen, H. J. Herrmann, and E. Guyon, J. Stat. Phys. 52, 237 (1988).

[10] A. Hansen and P. C. Hemmer, Phys. Lett. A 184, 394 (1994).

[11] V. I. Räisänen, M. J. Alava, and R. M. Nieminen, Phys. Rev. B 58, 14288 (1998).

[12] S. Zapperi, P. K. V. V. Nukala, and S. Simunovic, Phys. Rev. E 71, 026106 (2005).

[13] P. M. Duxbury, P. D. Beale, and P. L. Leath, Phys. Rev. Lett. 57, 1052 (1986).

[14] P. M. Duxbury, P. L. Leath, and P. D. Beale, Phys. Rev. B 36, 367 (1987).

[15] P. D. Beale and P. M. Duxbury, Phys. Rev. B 37, 2785 (1988).

[16] P. D. Beale and D. J. Srolovitz, Phys. Rev. B 37, 5500 (1988).

[17] P. K. V. V. Nukala and S. Simunovic, Eur. Phys. J. B 37, 91 (2004). 
[18] M. Sahimi and J. D. Goddard, Phys. Rev. B 33, 7848 (1986).

[19] M. Sahimi and S. Arbabi, Phys. Rev. B 47, 713 (1993).

[20] M. Minozzi, G. Caldarelli, L. Pietronero, and S. Zapperi, Eur. Phys. J. B 36, 203 (2003).

[21] G. Meacci, A. Politi, and M. Zei, Europhys. Lett. 66, 55 (2004).

[22] P. K. V. V. Nukala, S. Simunovic, and S. Zapperi, J. Stat.
Mech.: Theory Exp. P08001 (2004).

[23] G. G. Batrouni and A. Hansen, Phys. Rev. Lett. 80, 325 (1998).

[24] F. Reurings and M. J. Alava, e-print cond-mat/0401592.

[25] Removing the last event implies that the peak observed in Ref. [21] does not appear here. See also Ref. [12]. 